\title{
Quark-quark double scattering and modified (anti-)quark fragmentation functions in nuclei
}

\author{
Ben-Wei Zhang ${ }^{\mathrm{a}, \mathrm{b}, \mathrm{c}}$, Xin-Nian Wang ${ }^{\mathrm{d}}$ and Andreas Schäfer ${ }^{\mathrm{b}}$ \\ a Institute of Particle Physics, Huazhong Normal University, Wuhan, China \\ ${ }^{\mathrm{b}}$ Institut für Theoretische Physik, Universität Regensburg, Regensburg, Germany \\ ${ }^{\mathrm{c}}$ Cyclotron Institute, Texas A\&M University, College Station, TX, USA \\ ${ }^{\mathrm{d}}$ Nuclear Science Division, Lawrence Berkeley National Laboratory, CA, USA
}

\begin{abstract}
Quark-quark double scattering in eA DIS and its contribution to quark and antiquark fragmentation functions are investigated with the generalized factorization of the relevant twist-four processes in pQCD. It is shown that the resulting modifications to quark and anti-quark fragmentation functions are different. While the numerical size of these effects cannot be determined from pQCD, the structure of our result leads to a number of qualitative predictions for the relative size of the effect for different hadrons. These qualitative predictions agree with the multiplicity ratios for positive and negative hadrons as observed by HERMES.
\end{abstract}

Multiple scattering in a nuclear medium is a key process to understand how hard processes are modified in nuclei. Detailed experimental information, which is available for such diverse reactions as electron-nucleus collisions $(e+$ $A)$ in fixed target kinematics and hadron-nucleus collisions $(h+A)$ and nucleusnucleus collisions $(A+A)$ at RHIC or LHC is confronted by highly advanced theoretical descriptions $[1,2,3,4,5,6]$. Still, however, a truly comprehensive and reliable description of all phenomena has not been reached. In this contribution we discuss one element which should be contained in such an ultimate theoretical description.

We analyse [7] quark-quark double scattering in nuclei and consider the nuclear effects on the quark and anti-quark fragmentation functions (FF), using the generalized factorization theory by LQS [8] in perturbative QCD. We find that quark-quark double scattering will give different corrections to quark and anti-quark FF, and obtain qualitative predictions which are in agreement with quite remarkable observation by the HERMES Collaboration [9].

In general, there are two different kinds of double scattering with nuclear en- 


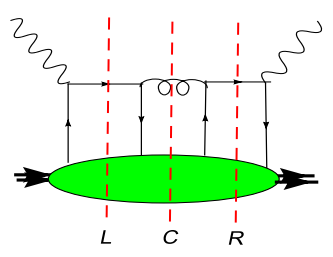

(a)

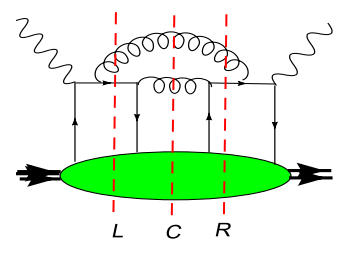

(b)

Fig. 1. Two typical diagrams for quark-quark double scattering with three possible cuts, central(C), left(L) and right(R): (a) lowest-order of quark-quark rescattering; (b)next-to-leading order quark-quark rescattering.

hancement in eA DIS: quark-gluon double scattering and quark-quark double scattering. Quark-gluon double scattering gives the dominant contribution to the leading quark energy loss and has been studied in detailed $[3,4,10]$, while quark-quark double scattering may mixes quark and gluon fragmentation functions and therefore gives rise to new nuclear effects, as pointed out by Wang and Guo [3]. This is illustrated in Fig. 1(a). For the central-cut diagram, the final parton is a gluon fragmenting into the hadron considered. For the leftcut or right-cut diagrams, however, the final fragmenting parton is a quark. Therefore, adding up the contributions of these three cut diagrams we will get a mixture of quark and gluon fragmentation functions. Moreover, we can see from the central cut of diagram (a) that the quark jet is converted into a gluon jet by annihilation with an anti-quark in the nucleus, which provides a mechanism to change the jet flavor [11].

For quark-quark double scattering, there are three cut diagrams in leadingorder as shown in Fig. 1(a), and 46 cut diagrams in next-to-leading order, see Fig. 1(b) [7]. By summing all these contributions and including virtual corrections we can rewrite the semi-inclusive tensor in terms of a modified fragmentation function taking into account quark-quark double scattering:

$$
\begin{array}{r}
\frac{d W_{\mu \nu}}{d z_{h}}=\sum_{q} \int d x f_{q}^{A}\left(x, \mu_{I}^{2}\right) H_{\mu \nu}^{(0)} \widetilde{D}_{q \rightarrow h}\left(z_{h}, \mu^{2}\right) \\
\widetilde{D}_{q \rightarrow h}\left(z_{h}, \mu^{2}\right)=D_{q \rightarrow h}\left(z_{h}, \mu^{2}\right)+\Delta \widetilde{D}_{q \rightarrow h}\left(z_{h}, \mu^{2}\right),
\end{array}
$$

where $H_{\mu \nu}^{(0)}$ is the partonic part for single scattering, $D_{q \rightarrow h}\left(z_{h}, \mu^{2}\right)$ are the leading-twist quark fragmentation functions in the vacuum, and the $\Delta \widetilde{D}_{q \rightarrow h}\left(z_{h}, \mu^{2}\right)$ are related to the twist-four parton correlation operators $[3,4,8]$ and could be approximated as:

$$
\begin{aligned}
& \Delta \widetilde{D}_{q \rightarrow h}\left(z_{h}, \mu\right) \approx \frac{2 \pi \alpha_{s} x_{B}}{x_{A} Q^{2}} \frac{2 C_{F}}{N_{C}}\left[D_{g \rightarrow h}\left(\frac{z_{h}}{z}\right)-D_{q \rightarrow h}\left(\frac{z_{h}}{z}\right)\right] C f_{\bar{q}}^{N}\left(x_{T}\right) \\
& +\int_{0}^{\mu^{2}} \frac{d \ell_{T}^{2}}{\ell_{T}^{2}} \frac{\alpha_{s}^{2} x_{B}}{x_{A} Q^{2}} \int_{z_{h}}^{1} \frac{d z}{z} D_{q \rightarrow h}\left(\frac{z_{h}}{z}\right) \frac{1+z^{2}}{(1-z)_{+}^{2}} \frac{C_{F}}{N_{C}}\left[1-e^{-x_{L}^{2} / x_{A}^{2}}\right] C f_{\bar{q}}^{N}\left(x_{T}\right)
\end{aligned}
$$




$$
-\int_{0}^{\mu^{2}} \frac{d \ell_{T}^{2}}{\ell_{T}^{2}} \frac{\alpha_{s}^{2} x_{B}}{x_{A} Q^{2}} \int_{0}^{1} d z D_{q \rightarrow h}\left(z_{h}\right) \frac{1+z^{2}}{(1-z)^{2}} \frac{C_{F}}{N_{C}}\left[1-e^{-x_{L}^{2} / x_{A}^{2}}\right] C f_{\bar{q}}^{N}\left(x_{T}\right) .
$$

Here $x_{A}=1 / m_{N} R_{A}, f_{q}^{A}(x)$ is the quark distribution inside a nucleus, $f_{\bar{q}}^{N}(x)$ is the anti-quark distribution inside a nucleon and $C$ is assumed to be a constant, $x_{T}=<k_{T}^{2}>/ 2 p^{+} q^{-} z$ and $k_{T}$ is the typical transverse momentum carried by the antiquark. If we consider the modification to an anti-quark FF all the calculations are the same with $q \leftrightharpoons \bar{q}$. Therefore, we have

$$
\frac{\Delta \widetilde{D}_{q \rightarrow h}\left(z_{h}, \mu^{2}\right)}{\Delta \widetilde{D}_{\bar{q} \rightarrow h}\left(z_{h}, \mu^{2}\right)} \approx \frac{f_{\bar{q}}^{N}\left(x_{T}\right)}{f_{q}^{N}\left(x_{T}\right)}<1
$$

HERMES [9] has measured the multiplicity ratio $R_{M}^{h}$, which represents the ratio of the number of hadrons of type $h$ produced per DIS event for a nuclear target of mass A to that from a deuterium target (D):

$$
R_{M}^{h}(z, \nu)=\frac{N_{h}^{A}(z, \nu)}{N_{e}^{A}(\nu)} / \frac{N_{h}^{D}(z, \nu)}{N_{e}^{D}(\nu)}
$$

with $N_{h}(z, \nu)$ the number of semi-inclusive hadrons in a given $(z, \nu)$-bin, and $N_{e}(\nu)$ the number of inclusive DIS positrons in the same $\nu$-bin.

In the constituent quark model, we have $p=u u d, \bar{p}=\bar{u} \bar{u} \bar{d}, \pi^{+}, \pi^{0}, \pi^{-}=$ $u \bar{d},(u \bar{u}-d \bar{d}) / \sqrt{2}, d \bar{u}$. Because the gluon FF for a positive hadron and its antiparticle are the same, and as we are interested only in the difference between the multiplicities of positive hadrons and negative hadrons, we neglect the contributions from gluon FF and simply assume:

$$
\Delta R_{M}^{h}(z, \nu) \equiv 1-R_{M}^{h}(z, \nu) \approx \frac{1}{N} \sum_{a} \Delta \widetilde{D}_{a \rightarrow h}(z, \nu)
$$

where $a$ is a constituent quark of hadron $h$, and $N$ is the number of these constituent quarks.

Therefore we obtain

$$
\begin{aligned}
& \Delta R_{M}^{\pi} \approx \frac{1}{2}\left(\Delta \widetilde{D}_{q \rightarrow h}(z, \nu)+\Delta \widetilde{D}_{\bar{q} \rightarrow h}(z, \nu)\right) \propto\left[f_{\bar{q}}^{N}\left(x_{T}\right)+f_{q}^{N}\left(x_{T}\right)\right] \\
& \Delta R_{M}^{p} \approx \Delta \widetilde{D}_{q \rightarrow h}(z, \nu) \propto f_{\bar{q}}^{N}\left(x_{T}\right), \Delta R_{M}^{\bar{p}} \approx \Delta \widetilde{D}_{\bar{q} \rightarrow h}(z, \nu) \propto f_{q}^{N}\left(x_{T}\right) \\
& \Delta R_{M}^{\pi^{+}}(z, \nu) \simeq \Delta R_{M}^{\pi^{-}} \simeq \Delta R_{M}^{\pi^{0}} \\
& \Delta R_{M}^{\bar{p}}(z, \nu)>\Delta R_{M}^{p}(z, \nu)
\end{aligned}
$$


Similarly we get

$$
\Delta R_{M}^{K^{-}}(z, \nu)>\Delta R_{M}^{K^{+}}(z, \nu), \quad \Delta R_{M}^{h^{-}}(z, \nu)>\Delta R_{M}^{h^{+}}(z, \nu) .
$$

The qualitative statements of Eq. (7), (8), and (9) agree with the observations made by the HERMES collaboration [9].

\section{Acknowledgements}

The authors thank Jian-Wei Qiu and Enke Wang for helpful discussion. This work was supported by Alexander von Humboldt Foundation, by BMBF, by NSFC under project No. 10405011, by MOE of China with project No. CFKSTIP-704035, the U.S. Department of Energy under Contract No. DEAC02-05CH11231, and by the US NSF under Grant No. PHY-0457265, the Welch Foundation under Grant No. A-1358.

\section{References}

[1] M. Gyulassy, I. Vitev, X. N. Wang and B. W. Zhang, Quark-Gluon Plasma 3, R. C. Hwa and X.-N Wang, Eds. (World Scientific, Singapore, 2003) .

[2] J. W. Qiu and G. Sterman, Int. J. Mod. Phys. E 12 (2003) 149.

[3] X. F. Guo and X.-N. Wang, Phys. Rev. Lett. 85, 3591 (2000); X.-N. Wang and X. F. Guo, Nucl. Phys. A 696, 788 (2001).

[4] B. W. Zhang and X.-N. Wang, Nucl. Phys. A 720, 429 (2003); B. W. Zhang, E. Wang and X. N. Wang, Phys. Rev. Lett. 93, 072301 (2004); Nucl. Phys. A 757, 493 (2005); B. W. Zhang, arXiv:nucl-th/0411076].

[5] R. Baier, D. Schiff and B. G. Zakharov, Annu. Rev. Nucl. Part. Sco. 50, 37 (2000).

[6] X. N. Wang, Phys. Lett. B 595, 165 (2004).

[7] B. W. Zhang, X. N. Wang and A. Schäfer, to be published.

[8] M. Luo, J. W. Qiu and G. Sterman, Phys. Rev. D 50 (1994) 1951; Phys. Rev. D 49, 4493 (1994).

[9] A. Airapetian et al. [HERMES Collaboration], Eur. Phys. J. C 20, 479 (2001) ; Phys. Lett. B 577, 37 (2003).

[10] E. Wang and X.-N. Wang, Phys. Rev. Lett. 89, 162301 (2002).

[11] W. Liu, C. M. Ko and B. W. Zhang, [arXive:nucl-th/0607047]. 